\title{
Cervical kyphotic deformity
}

\author{
Vincent C. Traynelis, MD \\ Department of Neurosurgery, Rush University Medical Center, Chicago, Illinois
}

$\mathrm{T}$ oBIN et al. share their experience with and technique of T-1 pedicle subtraction osteotomies (PSOs) for the treatment of significant fixed cervical deformities. ${ }^{3}$ Upper thoracic osteotomies limit risk to the vertebral artery and C-8 nerve root as compared to a C-7 PSO and still offer an opportunity to achieve substantial correction. The advantages of these procedures have been described by others as well.1,2

Cervical kyphotic deformities are heterogeneous in many regards. There is a spectrum of severity as well as diversity in the region producing kyphosis. A number of effective techniques may be safer than a PSO in a properly chosen patient or can add to the correction. For example, in their Case 1 (illustrated in their Fig. 6), the kyphotic deformity is from C-2 to C- 6 and the T-1 slope is fairly flat. A posterior release from C-2 to C-6 followed by an anterior release and correction would have corrected the kyphosis adequately. Although the final posterior instrumentation placement makes this a $540^{\circ}$ procedure, the complication risk and the total number of levels requiring arthrodesis are both less than necessary with a PSO and extension of the fusion caudally. The availability of several different operative procedures enhances the surgeon's ability to perform corrections with minimal complications, but it also increases the difficulty in choosing the proper surgical strategy.

The complication profile for all PSO procedures, including those in the upper thoracic spine, is robust. ${ }^{2}$ Nonunion is a particular issue that has resulted in the use of 4 rods by some surgeons. Tobin et al. utilized a fusion technique that involves the direct placement of bone on the dura mater and the off-label use of bone morphogenetic protein-2. This was effective in this small series, but there is concern that the dura could become calcified, which could make revision surgery more difficult.

A growing body of literature touts the benefits of aggressive normalization of a number of spinal parameters, especially as they relate to the sagittal plane. While there are patients who will benefit from extensive efforts to achieve correction, the correlation factor for many of the alignment/outcome relationships is only moderate. Each patient must be carefully assessed and all techniques considered before deciding on a PSO since, as physicians, we must first do no harm.

https://thejns.org/doi/abs/10.3171/2016.11.SPINE161033

\section{References}

1. Deviren V, Scheer JK, Ames CP: Technique of cervicothoracic junction pedicle subtraction osteotomy for cervical sagittal imbalance: report of 11 cases. J Neurosurg Spine 15:174-181, 2011

2. Theologis AA, Tabaraee E, Funao H, Smith JS, Burch S, Tay B, et al: Three-column osteotomies of the lower cervical and upper thoracic spine: comparison of early outcomes, radiographic parameters, and peri-operative complications in 48 patients. Eur Spine J 24 (Suppl 1):S23-S30, 2015

3. Tobin MK, Birk DM, Rangwala SD, Siemionow K, Schizas C, Neckrysh S: T-1 pedicle subtraction osteotomy for the treatment of rigid cervical kyphotic deformity: report of 4 cases. J Neurosurg Spine [epub ahead of print August 25, 2017. DOI: 10.3171/2016.8.SPINE121065]

\section{Disclosures}

Dr. Traynelis is a consultant for and holds a patent with Medtronic and has received institutional fellowship support from Globus.

\section{Response}

\section{Matthew K. Tobin, BS, ${ }^{1}$ Daniel M. Birk, MD, ${ }^{1}$ Shivani D. Rangwala, MD, ${ }^{1}$ Krzysztof Siemionow, MD, ${ }^{2}$ Constantin Schizas, MD, ${ }^{3,4}$ and Sergey Neckrysh, MD ${ }^{1}$}

Departments of ${ }^{1}$ Neurosurgery and ${ }^{2}$ Orthopaedic Surgery, University of Illinois at Chicago, Illinois; ${ }^{3}$ Spine Unit, Centre Hospitalier Universitaire Vaudois and University of Lausanne; and ${ }^{4}$ Orthopedic Spine Unit, Clinique Cecil Neuro, Lausanne, Switzerland

First, we thank Dr. Traynelis for his insightful comments regarding our paper. He brings warranted attention 
to the need for important decision making regarding the most appropriate surgical approach for patients with cervical deformity. The focus of our paper was not necessarily to compare PSO to other well-accepted methods of correction for cervical deformity, but rather to compare the efficacy of PSO performed at T-1 rather than at C-7 for specifically rigid cervical deformity. In our experience, PSO in patients with such deformities results in fewer complications (for example, nerve root injury, dysphagia, and so forth) than those in patients undergoing a combined anteroposterior release. In patients with nonrigid cervical kyphotic deformity, we routinely perform combined anteroposterior release procedures, and we agree that good spine reconstruction is often better with these approaches than with PSO in patients with nonrigid deformity.

Dr. Traynelis refers to our Fig. 6 as an example of the potential use of such a combined approach, which was, in fact, performed in our patient. Following a T-1 PSO, this patient underwent an anterior surgical approach as well, as demonstrated in Fig. 6. One important aspect to consider is whether previous posterior surgery has been performed, as was the case in our patient. She developed increased cervical deformity after undergoing occiput to C-6 posterior fusion, necessitating additional corrective surgery. In such revision patients, more aggressive posterior releases with or without the combination of an anterior approach may be necessary to achieve adequate correction, as demonstrated in our patient. We argue, though, that in patients who do require a PSO, surgeons should consider performing this procedure at $\mathrm{T}-1$ rather than $\mathrm{C}-7$ to eliminate the risks we outline when operating around $\mathrm{C}-7$.

Regarding the use of a 4-rod technique, a PSO in the thoracic spine offers the advantageous ability to use larger rods (5.5 vs $3.5 \mathrm{~mm}$ in the cervical spine), helping to strengthen the construct and eliminate nonunion or rod fracture. Furthermore, cervical and thoracic bone is very well vascularized, aiding fusion rates. Additionally, with regard to bone morphogenetic protein-2 (BMP-2) and placement of bone directly on the dura, we acknowledge that while their use was successful in our patients, our case series was small and the potential for complications in a larger patient series could become more apparent. However, these techniques are effectively used in other procedures, such as vertebral column resection, indicating that long-term complications are unlikely. We have not experienced issues using BMP-2 or placing bone directly on dura, but Dr. Traynelis makes an important point that doing so could make revision surgeries more complicated should calcification occur. This exposes an important area of study that should be investigated in a larger patient cohort.

We agree with Dr. Traynelis that sufficient preoperative planning is required to determine the most appropriate approach for each patient. Pedicle subtraction osteotomy and other more aggressive approaches are not suitable for all patients, but they are effective in certain patients. However, the level at which these procedures are performed must also be taken into consideration as operating at certain levels such as C-7 poses substantial risk to the patient and operating at alternate levels such as T-1 should be discussed when planning surgery for these patients. Lastly, we would like to point out that PSO is typically performed as a definitive (final) operation for patients in whom previous surgery has not been appropriately corrective. 